\title{
Magnetohydrodynamic Instabilities in a Simple Gasdynamic Mirror Propulsion System
}

\author{
William J. Emrich, Jr. ${ }^{\dagger}$ \\ NASA Marshall Space Flight Center, Huntsville, Alabama 35812 \\ and \\ Clark W. Hawk ${ }^{\ddagger}$ \\ University of Alabama at Huntsville, Huntsville, Alabama 35899
}

\begin{abstract}
The gasdynamic mirror has been proposed as a concept which could form the basis of a highly efficient fusion rocket engine. Gasdynamic mirrors differ from most other mirror type plasma confinement schemes in that they have much larger aspect ratios and operate at somewhat higher plasma densities. To evaluate whether a gasdynamic mirror could indeed confine plasmas in a stable manner for long periods of time, a small scale experimental gasdynamic mirror was built and tested. The objective of this experiment was to determine ranges of mirror ratios and plasma densities over which gasdynamic mirror could maintain stable plasmas. Theoretical analyses indicated that plasma magnetohydrodynamic instabilities were likely to occur during subsonic to supersonic flow transitions in the mirror throat region of the gasdynamic mirror. The experimental evidence based upon data derived from the Langmuir probe measurements seems to confirm this analysis. The assumption that a gasdynamic mirror using a simple mirror geometry could be used as a propulsion system, therefore, appears questionable. Modifications to the simple mirror concept are presented which could mitigate these MHD instabilities.
\end{abstract}

'Engineer, Propulsion Research Center, Member AIAA

${ }^{\ddagger}$ Professor, Propulsion Research Center, Fellow AIAA 


\section{Nomenclature}

Symbol Definition

A cross-sectional area

$A_{p c} \quad$ cross-sectional area of the plasma in the main chamber

$A_{p m} \quad$ cross-sectional area of the plasma at the mirror throat

$A^{*} \quad$ plasma cross-sectional area at which sonic flow occurs

$B \quad$ local magnetic field strength

$B_{p c} \quad$ magnetic field strength in the plasma at the center of the main chamber

$B_{p m} \quad$ magnetic field strength in the plasma at the mirror throat

c speed of sound

$\mathrm{I}_{\mathrm{sp}} \quad$ rocket specific impulse

$j_{\max } \quad$ number of points comprising a measured plasma density or temperature profile

K specific heat ratio

$l \quad$ position

$m \quad$ atomic weight of the plasma ions

$\dot{m} \quad$ mass flow rate

$M_{c} \quad$ chamber mach number

$n \quad$ plasma density

$\boldsymbol{P}_{\|} \quad$ component of pressure parallel to the magnetic field

$P_{\perp} \quad$ component of pressure perpendicular to the magnetic field

$P^{*} \quad$ pressure in nozzle throat at mach 1

$r \quad$ radius

$R \quad$ vacuum mirror ratio 


$\begin{array}{ll}R_{c} & \text { radius of curvature of the magnetic field } \\ R_{e} & \text { effective mirror ratio accounting for finite plasma pressure } \\ R_{u} & \text { universal gas constant } \\ t_{95} & \text { " } \mathrm{t} \text { " distribution } 95 \% \text { confidence limit } \\ T & \text { plasma ion temperature } \\ U & \text { uncertainty value } \\ <v> & \text { average particle velocity } \\ \beta & \text { ratio of plasma pressure to magnetic pressure } \\ \sigma & \text { standard deviation of the measured plasma density } \\ \Phi_{p c} & \text { plasma flux in central chamber } \\ \Phi_{p m} & \text { plasma flux at mirror throat } \\ \Psi & \text { ratio of plasma cross-sectional area at mirror throat to mirror throat cross- } \\ & \text { sectional area required for sonic flow }\end{array}$




\section{Introduction}

The large scale human exploration of the solar system will require the transportation of massive amounts of equipment and supplies over vast distances in space in relatively short periods of time. These requirements place a heavy burden on the rocket engines carrying out these missions. Studies have shown that chemical propulsion systems can perform manned missions only to the closest planets and even those only with great difficulty [1]. Manned flights beyond Mars do not appear to be possible with chemical systems regardless of the vehicle configuration. The inadequacy of traditional rocket engines is due to the fact that the energy density of chemical propellants is limited. This limitation puts restrictions on the maximum rocket engine efficiency and consequently limits the scope of the missions which can be executed. One type of propulsion system which appears to have the potential to provide the performance levels required for fast manned interplanetary travel is based on the use of fusion energy. Recent studies [2], [3] indicate that space vehicles employing fusion propulsion systems could be constructed with specific powers of 10 or greater. At these performance levels a one way mission to Mars could be accomplished in $\mathbf{4 0}$ days and a one way mission to Pluto could be accomplished in about 2 years. For comparison, a NASA study [4] concluded that a Mars mission using only chemical propulsion would require a 490 day round trip flight time (156 days outbound) and would require aerobraking at Mars to reduce fuel requirements to a manageable level.

One type of fusion reactor which has been proposed as the basis for a spacecraft propulsion system is the gasdynamic mirror (Figure 1). This type of device has been theorized to have fairly high specific powers and specific impulses as high as 100,000 sec [5]. Magnetic mirror reactors are well suited for rocket propulsion system applications because their open 


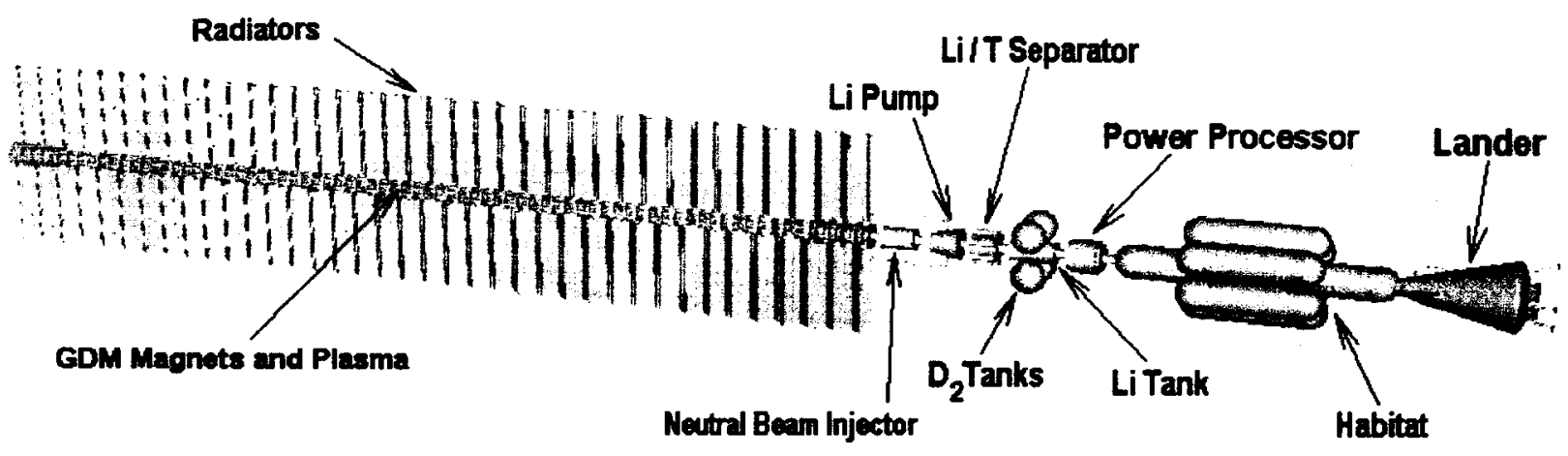

Figure 1 Interplanetary Vehicle Employing a Gasdynamic Mirror Propulsion System

ended linear geometry allows a natural path for the fusioning plasma exhaust. Mirror type fusion devices use a magnetic field line configuration called a "magnetic well" to confine the charged plasma particles. Plasma confinement has improved over the years with the newer mirror systems although this has generally come at the cost of increased complexity in the magnetic coil configurations at the mirrors. Mirror machines consist mainly of large multi-segment solenoids surrounding a vacuum chamber containing the plasma. A schematic diagram of a magnetic mirror machine illustrating a typical magnetic field line configuration is presented in Figure 2. The bulk of the fusion plasma is confined by magnetic fields generated within the central solenoid by a series of toroidal-shaped magnets. Stronger toroidal end magnets called mirror magnets prevent the plasma from escaping too quickly out the ends. The ratio of the maximum magnetic field strength in the mirrors divided by the minimum magnetic field strength in the central solenoid is called the mirror ratio $(R)$, and it is generally found that increasing the mirror ratio improves plasma confinement. Confinement is achieved in mirror machines because of constraints on particle motion imposed by the conservation of magnetic moment and the conservation of energy. Better confinement is especially desirable from a propulsion system 


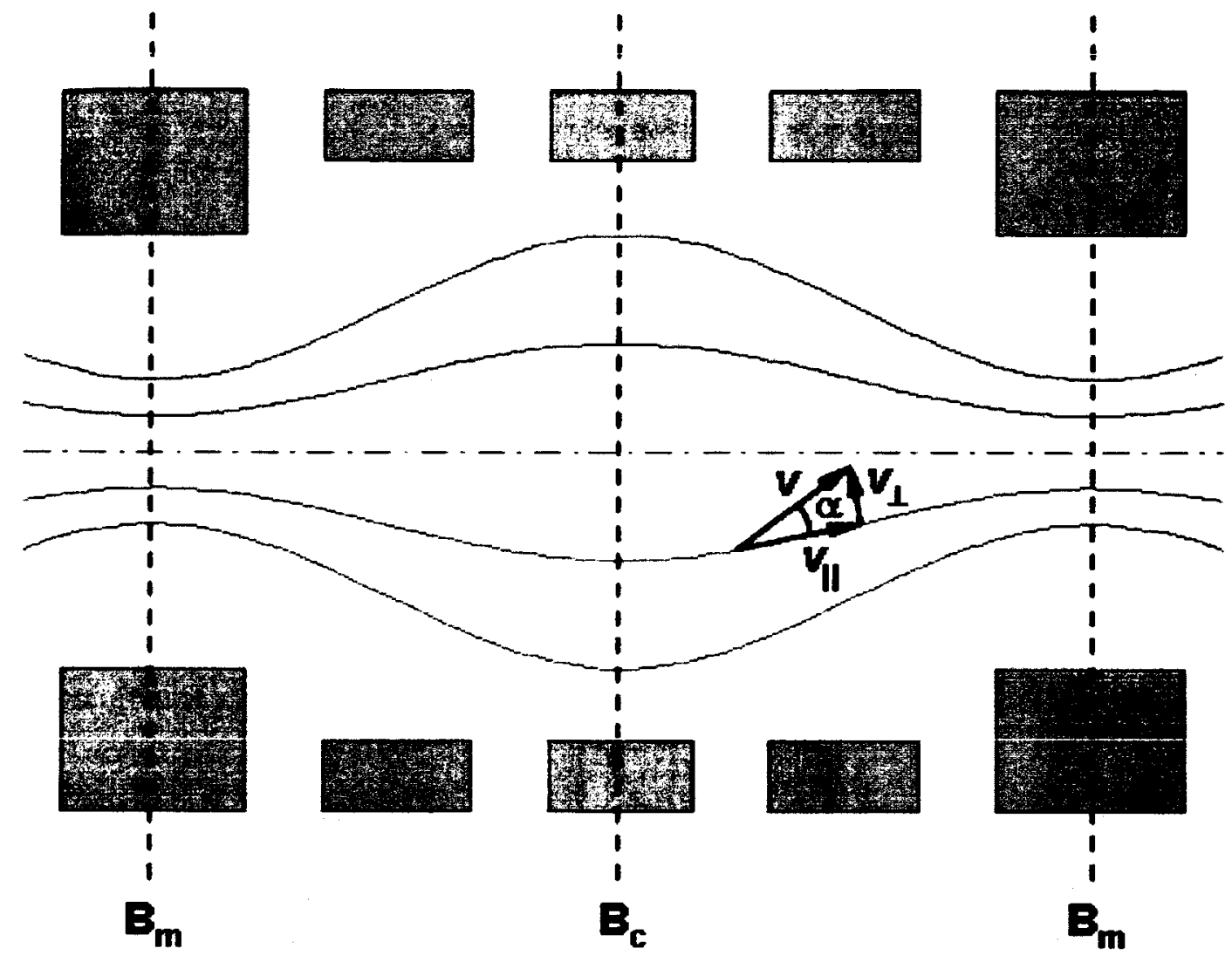

Figure 2 Particle Motion in a Mirror

standpoint since it translates directly into shorter system lengths and reduced mass. Previous analyses suggest that for gasdynamic mirror systems, the length necessary to achieve self sustained fusion is inversely proportional to the mirror ratio [6].

Raising the mirror ratio to high values to increase plasma confinement is not always effective, however, since high mirror ratios can also induce the occurrence of certain rather severe plasma instabilities in the device called magnetohydrodynamic or "MHD" instabilities. These instabilities are a result of convex curvature in the magnetic field lines with respect to the machine's centerline. In simple mirror machines, convex magnetic field line curvature occurs naturally in the main chamber region of the device, especially near the mirror magnets, and is accentuated by increases in the mirror ratio. The curvature of the magnetic field causes charge 
separations to occur between the electrons and the ions in the plasma. Electric fields induced by this charge separation cause uncontrollable ion drifts across the magnetic field lines which result in the appearance of plasma "flutes" which extend radially outward from the centerline of the plasma column (Figure 3). These flutes lead to a rapid loss of confinement as the plasma impacts the containment walls. This instability is also known as the interchange instability because a geometrical picture of the process reveals that there is an interchange between the magnetic flux originally outside the plasma boundary with plasma inside the plasma boundary. The degree to which the plasma may be made to remain stable is thought to be determined primarily by the relative density of plasma in regions of the device having convex "bad" magnetic curvature compared to the density of plasma in regions of the device having concave "good" magnetic curvature. Because subsonic to supersonic flow transitions cause the plasma density distribution

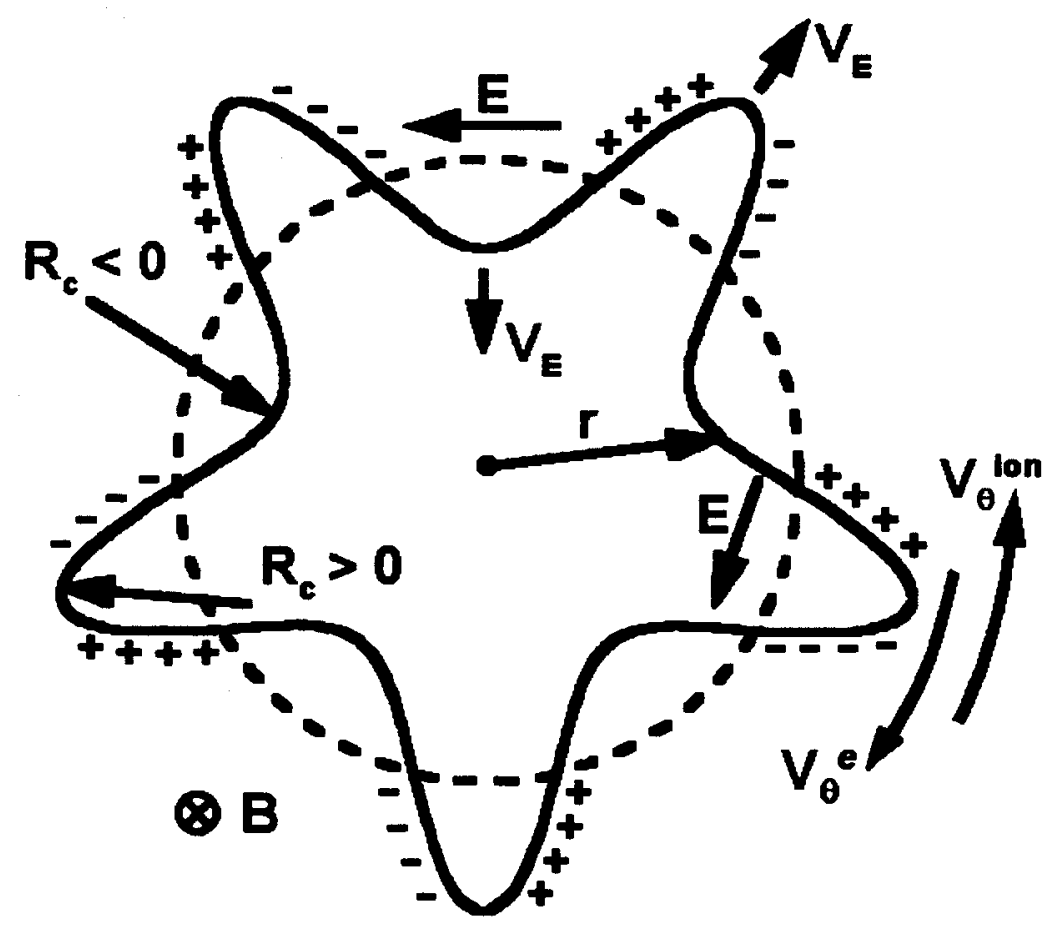

Figure 3 MHD Flute Instability 
to change drastically in regions of pronounced magnetic field curvature, a knowledge of flow transition effects with regard to plasma stability are crucial if magnetic mirror devices are to form the basis of rocket propulsion systems.

The plasma stability achieved with the gasdynamic trap at Novosibirsk, Russia [7] suggests that rocket engines based on similar designs should be feasible. Gasdynamic mirror devices have the advantage of being simple in construction and since they operate with plasma densities that are relatively high, they should enjoy good thrust to weight ratios. These advantages, however, are contingent upon the gasdynamic mirror operating in a stable manner under conditions in which self sustained fusion occurs (e.g. plasma densities $>10^{15} \mathrm{ion} / \mathrm{cm}^{3}$ and plasma temperatures $>10^{8} \mathrm{~K}$ ). To confirm the expected operational characteristics of a magnetic mirror based fusion propulsion system, a study was undertaken to examine theoretically and experimentally how plasma density variations between the convex and concave regions of magnetic field line curvature in a mirror device affect plasma stability.

\section{Magnetohydrodynamic (MHD) Instabilities}

The stability of plasmas in magnetic fields was first studied extensively by Rosenbluth and Longmire [8]. Their analysis established a fairly simple criterion whereby the stability of plasmas could be evaluated where:

$$
\text { Stability Factor }=\int \frac{P_{\|}(l)+P_{\perp}(l)}{r R_{c}(l) B^{2}(l)} d l \stackrel{\text { For Stability }}{<} 0 .
$$

In the above equation, $P_{\|}$and $P \perp$ are the parallel and perpendicular plasma pressures respectively, $r$ is the radius of the plasma column, $R_{c}$ is the local radius of curvature of a 
magnetic field line, and $B$ is the local magnetic field strength (Figure 3).

The gasdynamic mirror attempts to establish a stable plasma configuration by creating a long straight central region with little curvature in the magnetic field lines $\left(R_{c}=0\right)$, a short transition of bad curvature and a throat region of good curvature with significant plasma pressure. A previous analysis [9] of a gasdynamic mirror in which the velocity of the exit flow was limited to a low supersonic value implied that stable operation was possible. If the plasma exhaust jet is allowed to freely expand as would be the case for rocket operation, however, other results are possible. Applying equation (1) to the gasdynamic mirror using flow parameters and axial magnetic field profiles typical of the current experiment along with standard compressible flow equations, it is possible to draw a number of additional interesting conclusions regarding MHD stability in the GDM.

Figure 4 illustrates the axial variation of the stability factor given in equation (1) resulting from two almost identical supersonic main chamber plasma flow profiles. In one case the plasma flow is adjusted so that it remains slightly supersonic in the mirror throat resulting in a flow that remains supersonic in the diverging section of the magnetic mirror. In the other case the flow is increased slightly so that a sonic condition occurs in the mirror throat resulting in a flow transition to subsonic in the diverging section of the magnetic mirror. It will be observed that the transition to subsonic flow in the mirror throat results in a strong stabilizing effect on the plasma due to the high plasma density in this region of good magnetic curvature. Unfortunately, subsonic exhaust flows are not very desirable from a rocket engine efficiency point of view. This poor efficiency is due to the fact that $I_{s p}$ is proportional to the propellant exhaust velocity. If the plasma remains supersonic throughout the throat region and beyond, the plasma should still remain stable, though only marginally. This stability is the result of a fairly significant pressure 


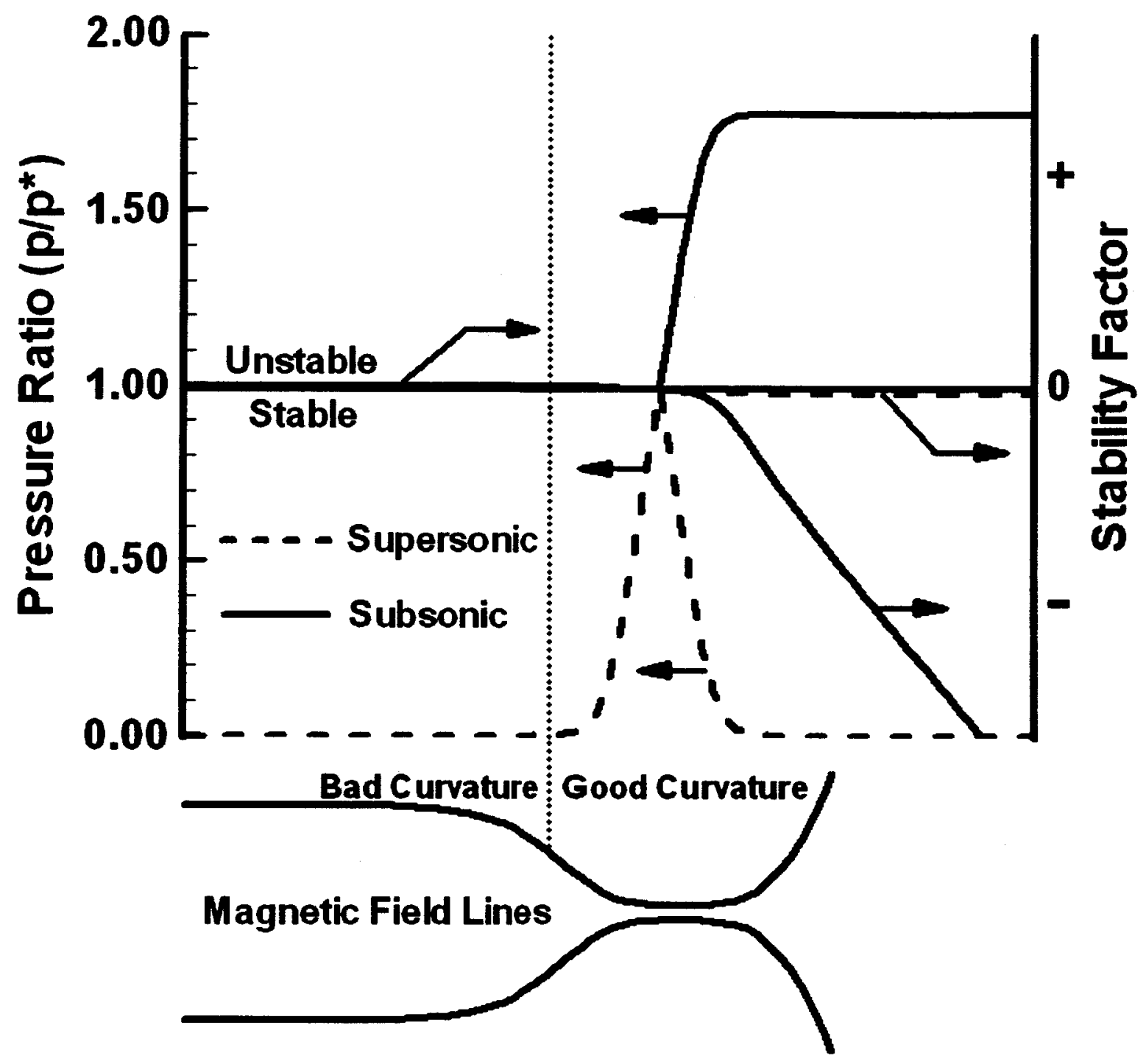

Figure 4 MHD Instability with Supersonic Chamber Flow

spike that occurs primarily in the region of good magnetic curvature near the throat of the gasdynamic mirror.

Figure 5 illustrates the axial variation of the stability factor given in equation (1) resulting from two almost identical subsonic main chamber plasma flow profiles. In one case the plasma flow is adjusted so that it remains slightly subsonic in the mirror throat resulting in a flow that remains subsonic in the diverging section of the magnetic mirror. In the other case the flow is increased slightly so that a sonic condition occurs in the mirror throat resulting in a flow 


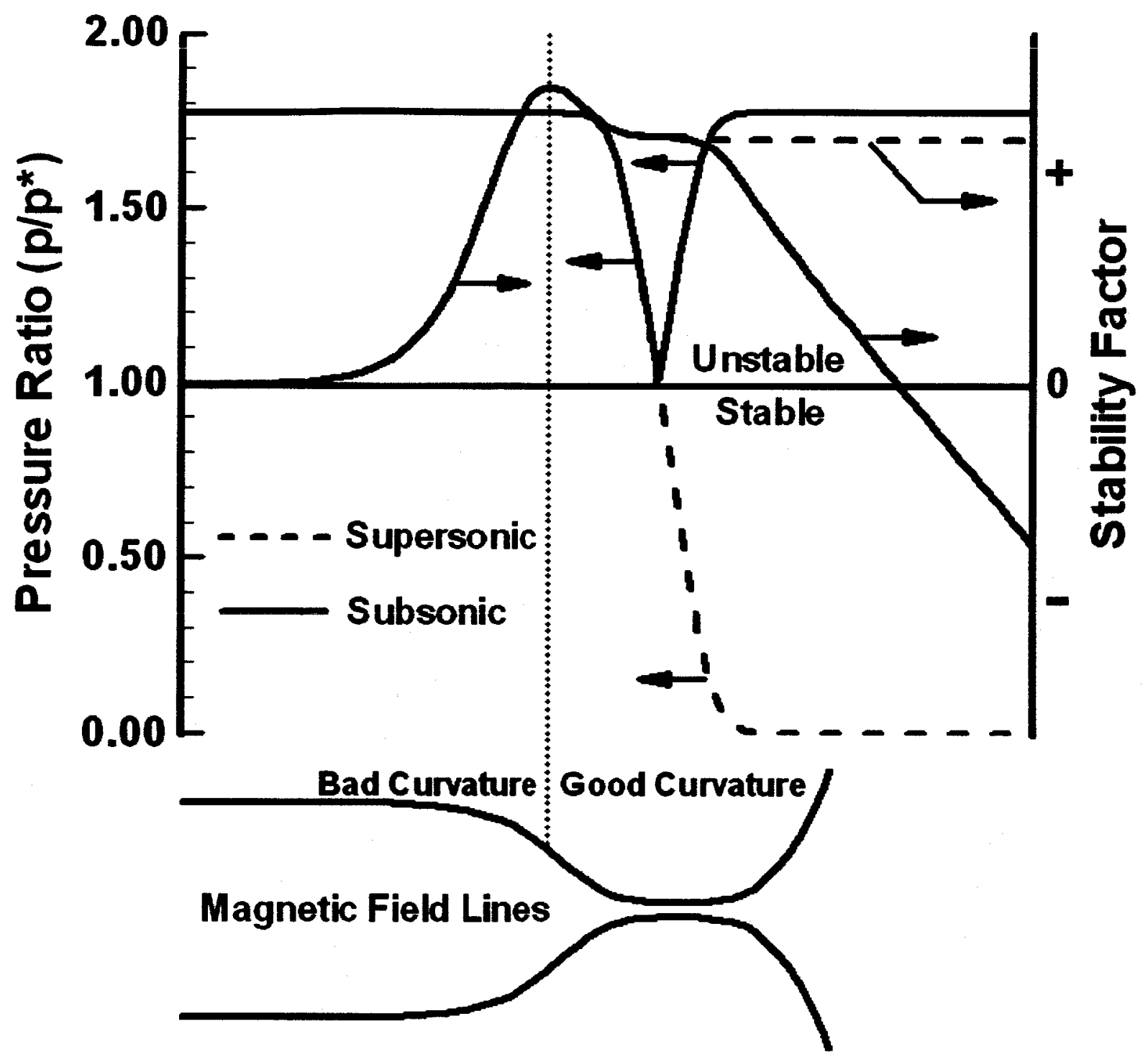

Figure 5 MHD Instability with Subsonic Chamber Flow

transition to supersonic in the diverging section of the magnetic mirror. It will be observed that a transition to supersonic flow in the mirror throat results in the plasma being highly unstable. The instability is caused by the plasma pressure decreasing rapidly throughout the diverging region of the magnetic mirror. In essence, the stabilizing effect of plasma in the region of good magnetic curvature near the mirror throat is overwhelmed by the destabilizing effect of the much higher density plasma in the converging section of the magnetic mirror where the magnetic curvature is bad. This situation is unfortunate since the normal flow distribution in rocket nozzles is 
generally similar to that just described. To compensate for this situation, it may be possible to externally inject high density, low temperature plasma into the diverging section of the magnetic mirror nozzle so as to raise the overall plasma density in this region of good magnetic curvature to the point where plasma stability may be restored. Whether this can be effectively done remains to be seen, however, and at this point such a remedy remains highly speculatively. Nevertheless, if such a procedure were effective, it would be highly beneficial to the engine system in that it would increase the thrust level and reduce the specific impulse. Since for many planetary missions, the specific impulse of the GDM is significantly higher than necessary, external plasma injection, if effective, would solve two problems at once by increasing plasma stability and providing a more optimum thrust and specific impulse combination.

If the flow is prevented from going supersonic in the throat, but instead remains subsonic throughout the flow regime, stability may be regained if the plasma does not detach from the magnetic field lines until it is relatively far downstream of the throat. This flow configuration would not require external plasma injection, but it also may not constitute a particularly desirable state of affairs. Since much of the exhaust will not be parallel to the direction of flight the engine will experience a considerable loss of propulsive efficiency.

\section{Experimental Setup}

In order to experimentally verify the theoretical predictions as previously outlined, a small gasdynamic mirror was built at the Marshall Space Flight Center (MSFC). Although the machine currently operates at temperatures far too low to initiate any fusion reactions, the geometric configuration is such that it should be able to answer many of the fundamental questions on plasma stability in the GDM. Since it was anticipated that a wide variety of plasma 
conditions would be eventually studied using the GDM device, much effort was expended in designing it to be as flexible and expandable as possible from the beginning. Table 1 presents the major physical characteristics of the GDM in its current configuration.

Table 1 GDM Experiment Physical Characteristics

\begin{tabular}{|l|l|}
\hline Description & Value \\
\hline Length (main chamber) & 2.0 meters \\
\hline Length (mirror chambers + one main chamber) & 2.5 meters \\
\hline Main chamber diameter & 20 centimeters \\
\hline Mirror chamber diameter & 6 centimeters \\
\hline Number of magnets per main chamber & 17 \\
\hline Number of magnets per mirror chamber & 12 \\
\hline Magnet current & up to 3000 amps \\
\hline Main chamber vacuum magnetic field & up to 0.35 tesla (centerline) \\
\hline Mirror chamber vacuum magnetic field & up to 2.05 tesla (centerline) \\
\hline Vacuum chamber length & 1.5 meters \\
\hline Vacuum chamber diameter & 1.2 meters \\
\hline Vacuum pumping speed & 800 liters/sec \\
\hline Microwave injector power & 1000 watts \\
\hline Microwave injector frequency & $2.45 \mathrm{GHz}$ \\
\hline
\end{tabular}

The gasdynamic mirror experiment was constructed in a modular fashion containing as a minimum two mirror segments and a main segment. This minimum configuration was the setup used in the present set of experiments. Provision has been made to allow for the addition of more main segments at a future date thus enabling the experiment to easily grow without the need for an extensive redesign. Each segment has been designed to operate independently of the others to the greatest extent practical. The water cooled copper magnets in each segment are 
controlled by their own power supplies and are fed by their own header systems. By independently controlling the power to each set of magnets, great flexibility is obtained with regard to being able to shape the magnetic fields within the device. Each of the mirror segments also has a small subsegment near the interface to the main chamber segment. This subsegment was designed so as to allow flexibility for future modifications to the mirror segments to enable the incorporation of such things as confinement enhancing devices, additional diagnostics, etc.

A closed loop cooling system runs cold water along a distribution system that runs along the entire length of the experiment. This water distribution system has a series of valves and quick disconnects to enable the segments to be easily broken apart when the experiment is reconfigured. The cooling system itself basically consists of a $\mathbf{3 0 0}$ gallon tank which is the reservoir for the cooling water, a water to water heat exchanger and a pump. Cold service water flows through the primary side of the heat exchanger and the water pump forces the cooling water from the reservoir through the experiment and finally to the secondary side of the heat exchanger.

The two mirror segments, which form the ends of the GDM are used to restrict the plasma loss from the system and have many common features. Each mirror segment has a vacuum pumping system which consists of a roughing pump and a turbopump, each has two power supplies. There are 12 magnets in each mirror, half of which are connected to each power supply. Limited field shaping is thus possible in the mirrors. Water boost pumps are used to circulate coolant through the magnets since the heating rates will be considerably larger in the mirror magnets as compared to the central segment magnets.

Although the mirror segments serve the same primary purpose, they have quite different secondary purposes. One mirror segment contains a large vacuum chamber. This chamber 
serves several purposes. First, the chamber can serve as a vacuum reservoir for the exhaust during high flow plasma tests. For these tests, the pumping system will be unable to maintain the desired downstream vacuum and the vacuum chamber will allow these tests to be conducted albeit for limited durations. Second, the vacuum chamber could also possibly serve as a means by which the plasma exhaust may be studied after the plasma leaves the GDM since many questions still exist as to how the plasma may be made to detach efficiently from the magnetic field lines after it leaves the device.

The other mirror segment contains the plasma injector and control circuitry. Currently, the plasma is generated and heated by a microwave device which is described below. The segment has been constructed to allow various types of plasma injectors to be used in the system. These may be mounted either before or after the mirror.

The central segment contains the bulk of the plasma in the gasdynamic mirror experiment. This segment is basically a $20 \mathrm{~cm}$ diameter vacuum tube containing various diagnostic ports and surrounded by 17 magnets. These magnets can produce central fields of up to a third of a tesla when operated at full current by the single power supply associated with this segment. The main chamber is attached to the mirror segments by short bellows connectors which permit a tight vacuum to be maintained along the length of the GDM. As was stated earlier, the design of the experiment allows multiple central segments to be easily incorporated into the device.

Control and monitoring functions for the Gasdynamic Mirror experiment are accomplished through the use of a computer program which checks a series of transducers that measure temperatures, flowrates, etc. in various parts of the device and also controls the operation of the various pumps and power supplies. 


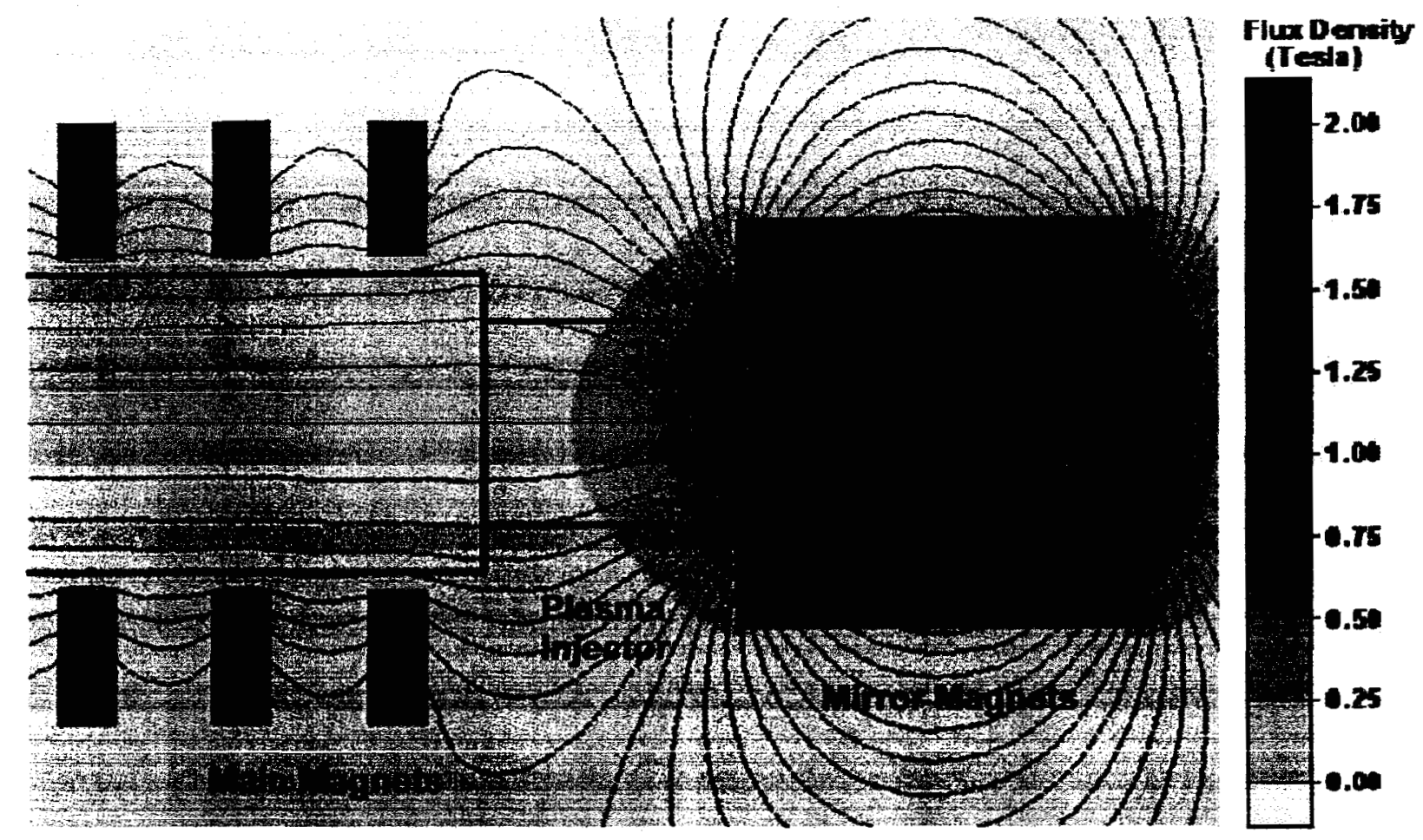

Figure 6 Magnetic Flux Density Map Near GDM Injector Segment

Figure 6 shows the magnetic flux density profile near the GDM injector segment. The magnetic field in the Gasdynamic Mirror is generated by a series of water cooled toroidal shaped magnets spaced periodically along the lengths of the main and mirror segments. The spacing between the magnets in the main chamber is large enough to allow diagnostic probes to be inserted between then, but not so large so as to create significant ripples in the magnetic field within the main plasma chamber. Even near the edge of the chamber where field variations are greatest, the ripple is only $3 \%$ to $4 \%$. A short distance away from the mirrors the magnetic field becomes quite flat. This flat profile is quite important in minimizing plasma magnetohydrodynamic instabilities, as will be discussed later.

Plasma heating in the Gasdynamic Mirror is accomplished through the use of a small plasma injector system located between the main chamber magnets and the mirror magnets. The 
purpose of the plasma injector is to introduce a gas (typically argon) into the GDM and heat it until it becomes a plasma. The injector operates by using a microwave antenna operating at 2.45 GHz to induce Electron Cyclotron Resonance Heating (ECRH) of the gas. The heating occurs when the magnetic field strength is such that the electrons resonate at the microwave injector frequency. This requirement imposes an operational constraint on the plasma injector system in that the magnetic field within the device must at some point correspond to the microwave injector frequency. Once the electrons have been heated by the microwaves, they will stream out of the injector and into the main plasma chamber in response to imposed magnetic field gradient. In doing so, they create an electric field which drags the ions along through a process called ambipolar diffusion. It is this process of ambipolar diffusion which increases the directed kinetic energy of the ions through energy transfer from the electrons.

\section{Experimental Results}

The experimental program for the Gasdynamic Mirror consisted of a series of plasma density and temperature measurements designed to define the stability limits of the device in terms of the vacuum chamber mirror ratio and the argon flow rate. To this end, the mirror ratio at the vacuum chamber end of the GDM was varied from 3 to 15 using argon flow rates fixed at either $2.5 \mathrm{sccm}$ or $6.0 \mathrm{sccm}$. The mirror ratio was varied solely by adjusting the magnetic field strength of the vacuum chamber segment mirror magnets. The main chamber magnets and the mirror magnets at the plasma injector end of the GDM were held fixed so as to maintain a constant injector segment mirror ratio of 9. Holding the injector segment mirror ratio constant was necessary because the plasma ion energy distribution varies as a function of the injector mirror ratio and a varying ion energy distribution could cause inconsistent results. 
From Langmuir probe measurements, average values for plasma density and electron temperature were calculated for the argon plasma column. These measurements were taken near the center of the main vacuum chamber. An analysis of these parameters yields information which can indicate the presence of plasma instabilities. Instabilities cause perturbations to occur in the plasma (flutes for MHD instabilities and turbulence for microinstabilities) which result in the plasma rapidly diffusing across the confining magnetic field lines. Since the confining capability of mirror machines largely depends upon the relative diameter of the throat area through which the plasma can escape, any perturbation which enhances cross field diffusion effectively enlarges the mirror throat area resulting in an increase in plasma loss rate. Because increases in the plasma loss rate limit the density that can be sustained in the device, instabilities will be detectable by drops in the plasma density and reduced confinement times. Table 2 presents typical values observed during the experiments for several plasma parameters.

Table 2 Typical Experimental GDM Plasma Parameters

\begin{tabular}{|l|c|}
\hline \multicolumn{1}{|c|}{ Parameter } & Value \\
\hline Plasma Density (ion $\left./ \mathrm{cm}^{3}\right)$ & $10^{13}$ \\
\hline Electron Temperature (eV) & 2 \\
\hline Beta & .003 \\
\hline Plasma Diameter in Main Chamber $(\mathrm{cm})$ & 4 \\
\hline Plasma Confinement Time (msec) & 10 \\
\hline $\mathrm{n} \tau$ (ion-sec/cm $\left.{ }^{3}\right)$ & $10^{11}$ \\
\hline Plasma Ion Collision Mean Free Path $(\mathrm{cm})$ & 0.1 \\
\hline
\end{tabular}




\section{Uncertainty Analysis}

Several scans were made at each mirror ratio and argon flow rate and the results combined to yield composite plasma density and electron temperature profiles. Because the positions of the density and temperature measurements varied from scan to scan, a linear interpolation was performed between adjacent measurement points within a profile so as to yield data values which could be placed on an evenly spaced grid. The data values thus placed on the grid from the various individual profiles were then averaged and standard deviations computed to yield the composite density or temperature profile. The average plasma density or electron

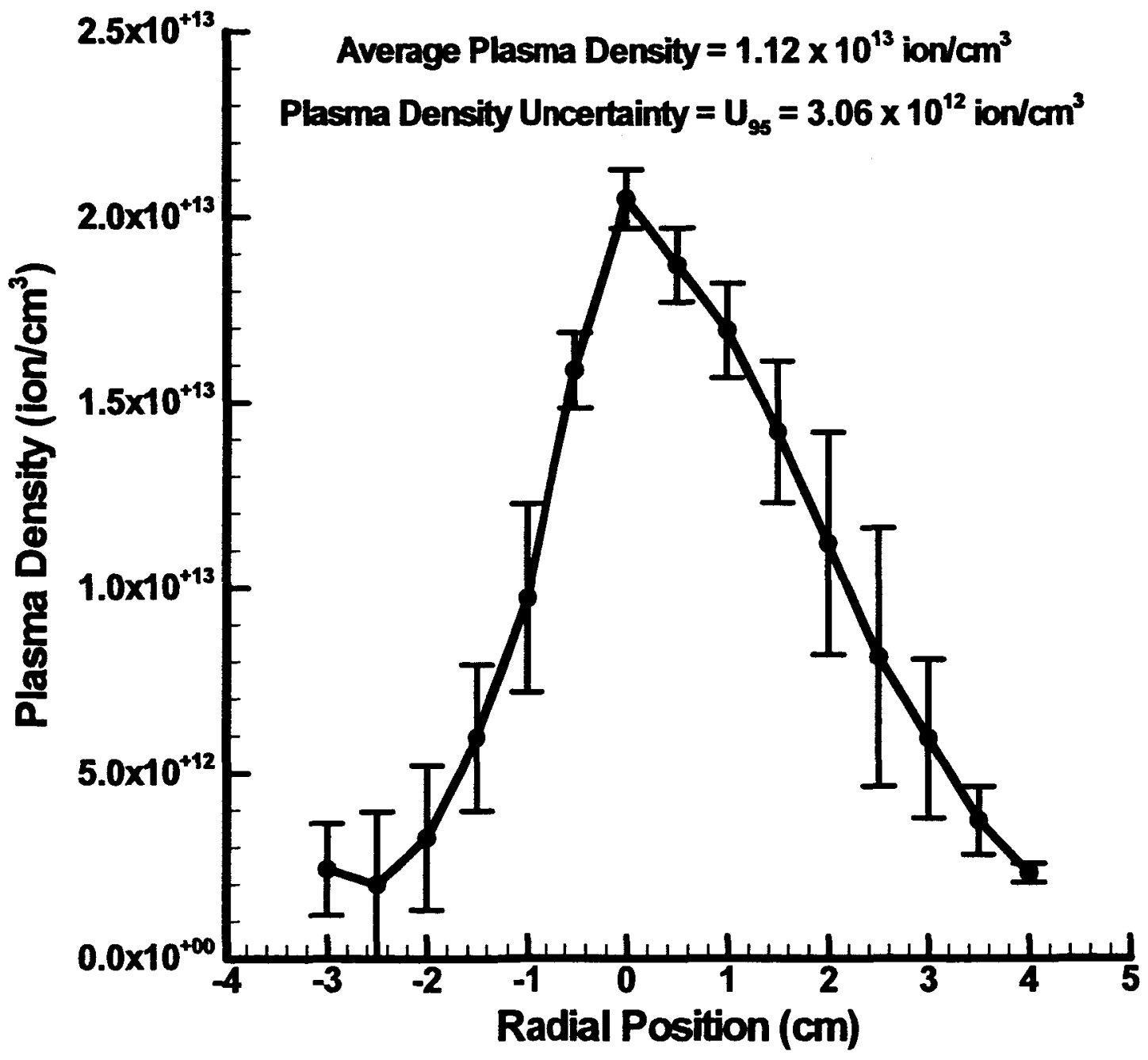

Figure 7 Averaged Langmuir Probe Data 
temperature for each individual profile was also calculated so as to permit the determination of the composite average values for those parameters along with their standard deviations for that particular combination of mirror ratio and argon flow rate. Figure 7 shows an example of an averaged plasma density profile.

\section{Data Averaging}

To perform the required plasma stability calculations, it was necessary to obtain profile averages of the plasma density and temperature. The average value for plasma density ( $n$ )was calculated by taking the composite plasma density profile and area weighting the pointwise

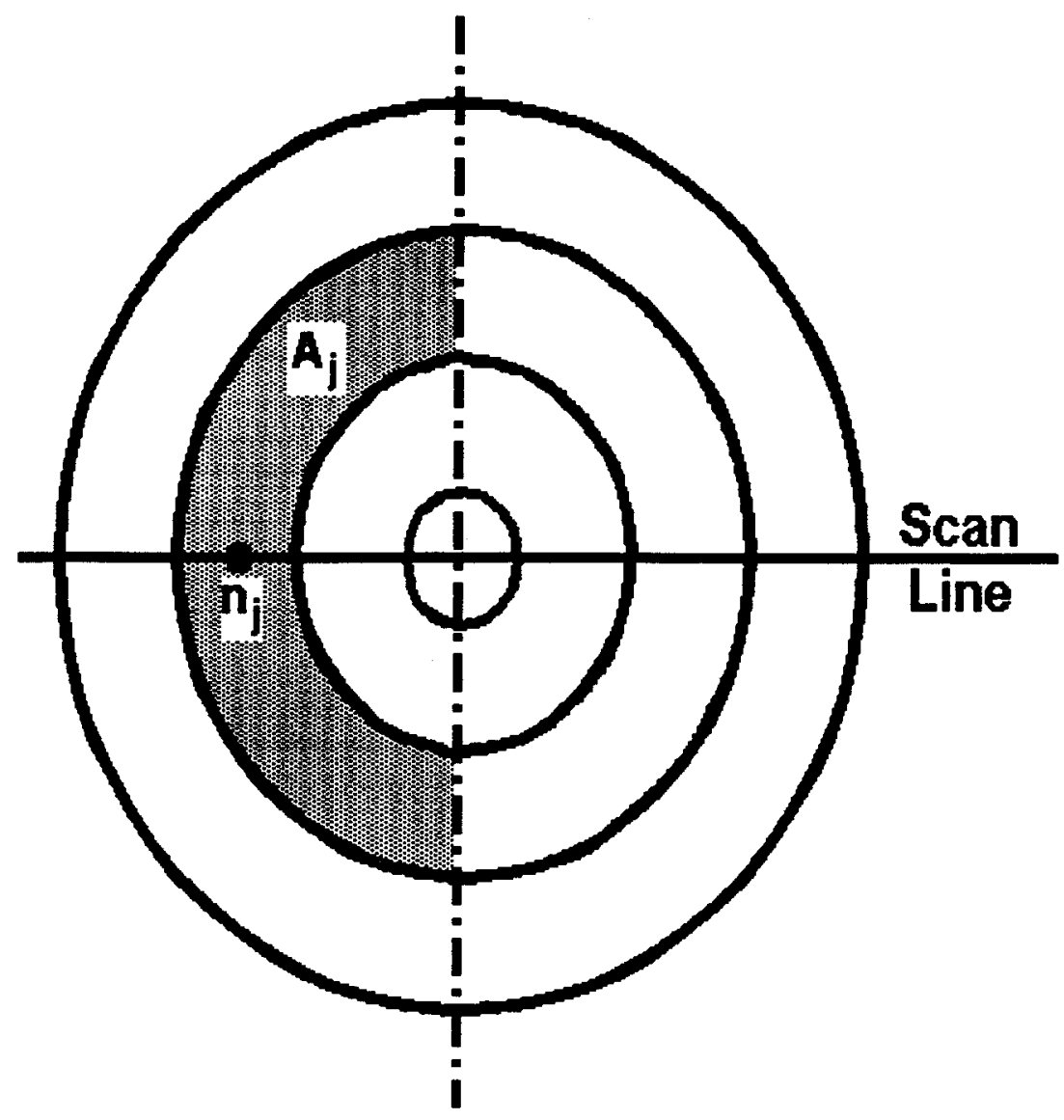

Figure 8 Plasma Areas Used in Data Averaging 
plasma densities centered around the point of maximum plasma density. The calculation proceeded using the following equation:

$$
n=\frac{\sum_{j=1}^{j_{\max }} A_{j} n_{j}}{\sum_{j=1}^{j_{\max }} A_{j}}=\frac{\sum_{j=1}^{j_{\max }} A_{j} n_{j}}{A} .
$$

where $n_{j}$ is the plasma density corresponding to plasma cross-sectional area $A_{j}$ (Figure 8). The plasma temperature $(T)$ was averaged in a similar manner except that it was weighted with both area and plasma density to obtain an energy averaged temperature. This averaging proceeded using a weighting equation of the form

$$
T=\frac{\sum_{j=1}^{j_{\max }} A_{j} n_{j} T_{j}}{\sum_{j=1}^{j_{\max }} A_{j} n_{j}} .
$$

The uncertainties $(U)$ in the average plasma density and temperature were calculated by using the general uncertainty equation [10] which when applied to the present situation yields for the uncertainty in plasma density

$$
U_{n}^{2}=\sum_{j=1}^{j_{\max }}\left[\left(\frac{\partial n}{\partial A_{j}}\right)^{2} U_{A_{j}}^{2}+\left(\frac{\partial n}{\partial n_{j}}\right)^{2} U_{n_{j}}^{2}\right]=\sum_{j=1}^{j_{\max }}\left(\frac{A_{j}}{A}\right)^{2} U_{n_{j}}^{2}
$$

For the uncertainty in the electron temperature the uncertainty equation is given by 


$$
\begin{aligned}
U_{T}^{2}=\sum_{j=1}^{j_{\max }}\left[\left(\frac{\partial T}{\partial A_{j}}\right)^{2} U_{A_{j}}^{2}+\left(\frac{\partial T}{\partial n_{j}}\right)^{2} U_{n_{j}}^{2}+\left(\frac{\partial T}{\partial T_{j}}\right)^{2} U_{T_{j}}^{2}\right] \\
=\sum_{j=1}^{j_{\max }}\left\{\left[\frac{\left.A_{j} T_{j}\left(\sum_{k=1}^{j_{m a x}} A_{k} n_{k}\right)-A_{j}^{2} n_{j} T_{j}\right]^{2} U_{n_{j}}^{2}+\left(\frac{A_{j} N_{j}}{\left.\sum_{k=1}^{j_{\max }} A_{k} A_{k} n_{k}\right)^{2}} U_{T_{j}}^{2}\right\}}{}\right.\right.
\end{aligned}
$$

The uncertainties in plasma density and electron temperature in equations (4) and (5) represent the $95 \%$ confidence limits on the respective measurements and were calculated from the standard deviations as follows

$$
U_{n}=\frac{\sigma_{n} t_{95}}{\sqrt{j_{\max }}}, \quad U_{T}=\frac{\sigma_{T} t_{95}}{\sqrt{j_{\max }}}, \quad U_{A} \approx 0\left(\text { small compared to } U_{n} \text { and } U_{T}\right)
$$

\section{MHD Stability Results}

Earlier it was theorized that MHD plasma instabilities should occur during subsonic to supersonic flow transitions in the mirror throat region of the GDM. These instabilities result in a loss of plasma confinement and are indicated by decreases in measured plasma density. As the mirror ratio is increased, the throat area through which the plasma flows decreases. At a given flow rate a mirror ratio is eventually reached where the flow goes sonic at the throat resulting in supersonic flow downstream. This mirror ratio is the maximum mirror ratio under which stable operation is possible and yields the maximum plasma density possible for that configuration.

To calculate the mirror throat mach number, the flow velocity $\langle v\rangle$ in the main chamber is first determined from the continuity equation 


$$
\langle v\rangle=\frac{\dot{m}}{n A_{p c}} .
$$

In the above equation, the flow rate $(\dot{m})$ is that which was set during the experiment and the plasma density $(n)$ and plasma main chamber cross-sectional area $\left(A_{p c}\right)$ are derived from the Langmuir probe measurements. The plasma cross-sectional area was calculated on the basis of the diameter of the plasma column within which the data was deemed to be valid. Under the test conditions used in this study, the plasma column diameter was about $4 \mathrm{~cm}$ with data outside this range showing no clear probe characteristic. The plasma sonic velocity was determined from the equation

$$
c=\sqrt{\frac{K R_{u} T}{m}} .
$$

The temperature ( $T$ ) in equation (8) was obtained from Langmuir probe measurements and is technically the electron temperature. It is assumed for this calculation, however, that the collisionality between the electrons and ions is such that thermodynamic equilibrium has been reached and that the temperatures for the two species can be taken to be the same. This assumption should be quite valid since with an ion mean free path of about $0.1 \mathrm{~cm}$, the ions will suffer hundreds of collisions with electrons before they reach the Langmuir probe which is located near the center of the GDM. The mach number in the main chamber can be calculated by

$$
M_{c}=\frac{\langle v\rangle}{c} .
$$

The chamber mach number calculated in equation (9) is then used in the isentropic compressible flow equation for simple area change [11] to determine the plasma cross-sectional area ratio 
required to achieve sonic flow at the mirror throat

$$
\frac{A_{p c}}{A^{*}}=\frac{1}{M_{c}} \sqrt{\left[\frac{2}{K+1}\left(1+\frac{K-1}{2} M_{c}^{2}\right)\right]^{\frac{k+1}{k-1}}} .
$$

The actual plasma cross-sectional area ratio at the mirror throat is estimated by noting that because of the conservation of magnetic flux lines

$$
\Phi_{p c}=\Phi_{p m} \Rightarrow B_{p c} A_{p c}=B_{p m} A_{p m} \Rightarrow \frac{A_{p c}}{A_{p m}}=\frac{B_{p m}}{B_{p c}}=R
$$

The results obtained from equations (10) and (11) may be combined to obtain a relation which yields the ratio of the actual area of the plasma at the mirror throat to the area of the throat which would be required to achieve sonic flow

$$
\Psi=\frac{A_{p m}}{A^{*}}=\frac{1}{R} \frac{A_{p c}}{A^{*}}=\frac{1}{R M_{c}} \sqrt{\left[\frac{2}{K+1}\left(1+\frac{K-1}{2} M_{c}^{2}\right)\right]^{\frac{k+1}{k-1}}}
$$

The uncertainty in the measured values for $\Psi$ may be calculated by first determining the uncertainty in the measured mach number by using equations (7), (8), and (9) in the general uncertainty equation. The resulting relation is then given by:

$$
U_{M}^{2}=\left(\frac{\partial M}{\partial n}\right)^{2} U_{n}^{2}+\left(\frac{\partial M}{\partial T}\right)^{2} U_{T}^{2}=\frac{\dot{m}^{2}}{K R_{u} A^{2} n^{2} T}\left(\frac{U_{n}^{2}}{n^{2}}+\frac{U_{T}^{2}}{4 T^{2}}\right)
$$

The uncertainty in $\Psi$ can now determined by again applying the general uncertainty equation using the relations described by equations (12) and (13). 


$$
U_{\Psi}^{2}=\left(\frac{\partial \Psi}{\partial M}\right)^{2} U_{M}^{2}=\frac{2 \sqrt{2}\left(M^{2}-1\right) \sqrt{\left(1+\frac{K-1}{2} M^{2}\right)^{\frac{K+1}{K-1}}}}{R M^{2}\left[2+(K-1) M^{2}\right] \sqrt{K+1}} U_{M}^{2} .
$$

The uncertainty term associated with the mirror ratio has been neglected in equation (14) since its uncertainty is small compared to the uncertainties in plasma density and electron temperature.

It should be noted that the mirror ratio used in equation (12) is the effective mirror ratio which the plasma sees, not the vacuum mirror ratio which is only a ratio of the mirror to central magnetic field strengths. The effective mirror ratio takes into account the effects of plasma pressure and is related to the vacuum mirror ratio by the following relation [12]

$$
R_{e}=\frac{R}{\sqrt{1-\beta}}
$$

If the plasma pressure is considerably less than the magnetic pressure $(\beta \ll 1)$ the two values differ by only a small amount and the error introduced by using only the vacuum mirror ratio in the calculations is small. Since the plasma should remain stable if $M_{t}$ is less than one (all subsonic flow), it follows that values of $A_{p m} / A^{*}>1$ must be maintained if stable plasma conditions are to be achieved. To confirm this conjecture, a set of measurements covering mirror ratios between 3 and 15 was performed at an argon flow rate of $6.0 \mathrm{sccm}$. From Figure 9, which is a plot correlating plasma density with mirror ratio and $\mathrm{A}_{\mathrm{pm}} / \mathrm{A}^{*}$, it is apparent that a fairly significant decrease in plasma density seems to occur between mirror ratios of 10 and 15 . Since the plasma flow transitions to supersonic in this mirror ratio regime also, it was strongly suspected that MHD instabilities were present. This suspicion was bolstered by the fact that the 


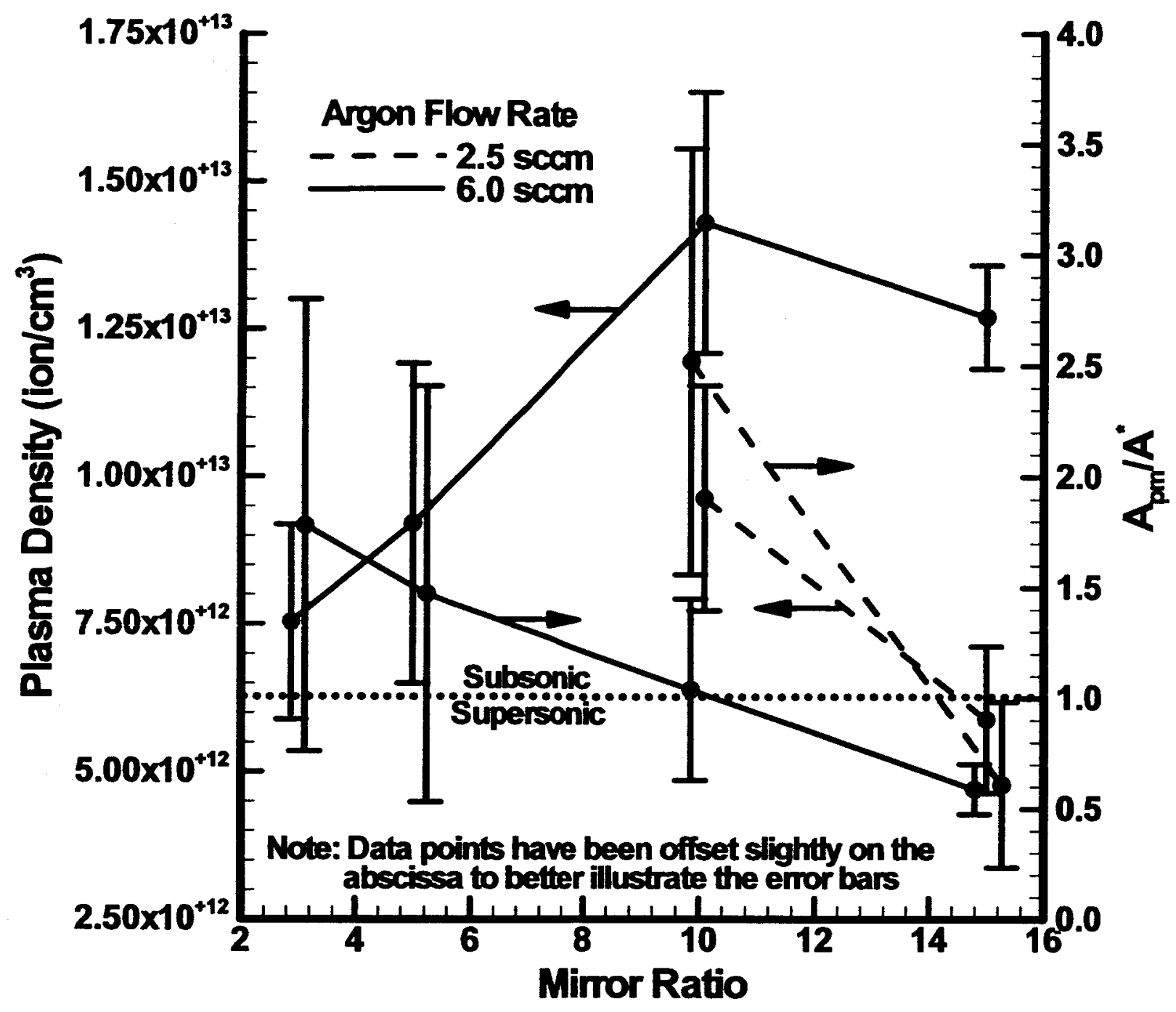

Figure 9 Measured Mirror Ratio Effects on Plasma Density

growth rate of MHD instabilities under normal experimental conditions was found to be much faster than the loss rate of plasma from the device. The growth rate $(\gamma)$ of the MHD instabilities was determined using a procedure described in [13] and is based upon an analysis of the plasma drift acceleration in curved magnetic fields. This plasma acceleration yields a growth rate of the form:

$$
\gamma=\sqrt{\frac{\left(v_{\perp}^{2} / 2+v_{I}^{2}\right)}{R_{c}} k_{y} .}
$$


Using parameter values characteristic of the GDM experiments at the point of maximum "bad" curvature yields results which indicate that MHD instabilities will begin to manifest themselves in about $5 \times 10^{-4} \mathrm{sec}$. Since the confinement time of plasma particles in the GDM was measured to be about $0.01 \mathrm{sec}$., it was concluded that MHD disruptions were the likely cause of the observed plasma density decreases. Other experimental runs to confirm the presence of MHD instabilities were performed under different conditions using an argon flow rate of $2.5 \mathrm{sccm}$. These runs which concentrated just on the subsonic to supersonic flow transition region showed a behavior similar to that observed at the higher flow rate. Similar drops in plasma density at certain critical values of $R$ have also been observed in Russian experiments with the gasdynamic trap at Novosibirsk [14] although it was impossible to confirm from the data presented that the plasma density drops occurred at the point of subsonic to supersonic transition.

\section{Conclusions}

The results of the Gasdynamic Mirror propulsion experiment indicate that MHD instabilities are likely to occur as a result of subsonic to supersonic flow transitions in the plasma exhaust stream. These instabilities result in a loss of plasma confinement and would almost certainly prevent the initiation of fusion reactions. As a result, the assumption that a gasdynamic mirror using a simple mirror geometry could be used as a propulsion system appears to be questionable. To overcome the stability problems in a gasdynamic mirror some sort of modification to the simple mirror concept will be required. Since the stability problem appears to be associated with the low plasma density associated with the transition to supersonic flow, it may be possible to stabilize the plasma through cold plasma injection into the hot plasma exhaust stream downstream of the mirror throat. This plasma injection would raise the plasma density in 
the regions of good magnetic curvature (the magnetic mirror nozzle throat and diverging section) to the point that the stability criterion as presented by equation (1) would be satisfied. The plasma injection would also help to reduce the inherently high specific impulse of the GDM and at the same time to increase its thrust to weight level to values more appropriate for solar system travel. Whether it is possible to achieve these results from a practical standpoint remains to be seen, however, and further work will be required.

\section{Acknowledgments}

This work was financially supported by NASA. The authors are grateful to Terry Kammash of the University of Michigan for the many useful discussions which occurred during the course of this work. We wish to also thank the many others at the Marshall Space Flight Center who participated in the construction of the gasdynamic mirror experiment and who contributed materially to the success of this work. 


\section{References}

1. Schulze, N., "Fusion Energy for Space Missions in the $21^{\text {st }}$ Century", NASA TM4298, Chapter 2, (Aug. 1991).

2. Kammash, T. and Emrich, W., "Interplanetary Missions with the GDM Propulsion System," STAIF-98, pages 1145-1150, (1998).

3. Emrich, W. and Kammash, T., "Performance Optimization of the Gasdynamic Mirror Propulsion System," STAIF-00, pages 1420-1424, (2000).

4. MSFC SEI Technical Study Team, "Space Exploration Initiative - Mars Transportation System Nuclear Thermal Rocket Propulsion Application (Addendum)", June 1992.

5. Kammash, T. and Lee, M. J., "A Gasdynamic Fusion Propulsion System for Space Exploration," Journal of Propulsion and Power, Vol 11, No. 3, page 544, (1995).

6. ibid

7. Bagryanskij, P. A., Ivanov, A. A., Klesov, V. V., et al., "First Experiments on the Gasdynamic Trap," Nuclear Fusion Supplement, Vol. 3, pages 467-476, (1987).

8. Rosenbluth, M. N. and Longmire, C. L., "Annals of Physics", 1, 120-140 (1957).

9. Nagornyj, V. P., Ryutov, D. D., and Stupakov, G. V., "Flute Instability of Plasma in a Gas-Dynamic Trap," Nuclear Fusion, Vol 24, No. 11, (1984), pages 1421-1438.

10. Coleman, H., and Steele, W., "Experimentation and Uncertainty Analysis for Engineers," John Wiley \& Sons, Inc., New York, NY, ISBN 0-471-12146-0, page 49, (1999).

11. Anderson, J. D., "Modern Compressible Flow: with Historical Perspective", $2^{\text {nd }}$ ed., McGraw-Hill, Inc., New York, ISBN 0-07-001673-9, page 155, (1990).

12. Kammash, T., "Fusion Reactor Physics," Ann Arbor Science Publishers, Inc., Ann 
Arbor, Michigan, ISBN 0-250-40076-6, page 333, (1975).

13. Miyamoto, K., "Plasma Physics for Nuclear Fusion," The MIT Press, Cambridge, MA, ISBN 0-262-13145-5, page 478, (1980).

14. Ivanov, A. A., et al., "Experimental Study of Curvature Driven Flute Instability in the Gasdynamic Trap," Physics of Plasmas, Vol. 1, No. 5, pages 1529 - xxxx, (1994) 